\title{
Algorithm for Target Recognition Based on Interval-Valued Intuitionistic Fuzzy Sets with Grey Correlation
}

\author{
Xuan Huang, ${ }^{1,2}$ Lihong Guo, ${ }^{1}$ Jiang $L i,{ }^{1}$ and Yang $Y u^{1}$ \\ ${ }^{1}$ Changchun Institute of Optics, Fine Mechanics and Physics, Chinese Academy of Sciences, Changchun 130033, China \\ ${ }^{2}$ University of Chinese Academy of Sciences, Beijing 130039, China \\ Correspondence should be addressed to Lihong Guo; guolh@ciomp.ac.cn
}

Received 2 January 2016; Revised 30 March 2016; Accepted 21 April 2016

Academic Editor: Anna M. Gil-Lafuente

Copyright (c) 2016 Xuan Huang et al. This is an open access article distributed under the Creative Commons Attribution License, which permits unrestricted use, distribution, and reproduction in any medium, provided the original work is properly cited.

\begin{abstract}
In order to improve exact recognition ratios for aerial targets, this paper presents a novel algorithm for target recognition based on interval-valued intuitionistic fuzzy sets with grey correlation. Drawbacks of some previously proposed methods are analyzed, and then a novel algorithm is presented. Recognition matrix of an aerial target is established first. Every entry associated with the matrix is an interval-valued intuitionistic fuzzy number, which is composed of interval-valued membership and nonmembership, representing the relation of the target to one category in terms of one characteristic parameter. Then grey correlation theory is used to analyze the recognition matrix to obtain the grey correlation degree of this unknown target to every category. 200 sets of target recognition data are used to compare the proposed algorithm with traditional methods. Experimental results verify that the correct recognition ratio can be up to $99.5 \%$ that satisfies the expectations, which shows the proposed algorithm can solve the target recognition problems better. The proposed algorithm can be used to solve the uncertain inference problems, such as target recognition, threat assessment, and decision making.
\end{abstract}

\section{Introduction}

Target recognition is the recognition and classification for the identity and characteristics of one target. Typically, it is in the first class level of JDL information fusion model. Whether the ground defense systems can recognize aerial targets quickly, reliably, and accurately, would directly affect the situation evaluation and threat assessment and finally has an influence on the allocation of defense power.

Target recognition is to confirm the category and identity of an unknown target according to its feature parameters. Due to the uncertainties in feature values, most of target recognition methods were developed typically based on the uncertainty theory, for example, D-S evidence $[1,2]$, Bayesian inference [3, 4], attribute measure [5], and fuzzy set [6]. However, these methods describe uncertainty information with drawbacks that resulted in the errors. Also, they may highly depend on the a priori knowledge. Therefore these methods may lead to low recognition accuracy in inference results.

Correlation analysis is a method that typically has a low requirement in the size of samples. It does not need any classical distribution laws in computation. Its results can match quite well the qualitative analysis. Therefore applying grey correlation theory in target recognition can guarantee the accuracy and real-time characteristics. In $[7,8]$, grey correlation analysis is used for target recognition by adopting the normalization of feature parameters to construct grey correlation coefficients and then giving a grey correlation sequence. The results show that the fairly accurate recognition ratios are obtained. But this method needs to compare the unknown target with the data chosen from the database. If some data in the database is not accurate enough, the recognition results would be incorrect.

In recent times, intuitionistic fuzzy set (IFS) $[9,10]$ is widely used in various areas, such as multiple feature decisions [11], information fusion [12], and image processing [13]. On the basis of Zadeh's fuzzy set (ZFS), nonmembership function is added in IFS, such that the utility of membership, nonmembership, and intuitionistic exponential can, respectively, signify the supportive, objective, and neutral characteristics. Thus IFS is able to describe the uncertainty information 
more flexibly and superiorly. In [14], IFS is introduced to accomplish target recognition through establishing a series of intuitionistic fuzzy inference rules. While IFS has quite high confidence, the issue becomes significantly difficult if there are numerous feature parameters. The likelihood of "combination explosion" enables the process complicated and impractical on real-world problems.

Due to the complexity and uncertainty in practice, the values of membership and nonmembership mostly could not be represented by the real values. Consequently Atanassov extended the intuitionistic fuzzy sets to the interval-valued intuitionistic fuzzy sets $[15,16]$, using an interval to express the membership and nonmembership. The nature of uncertainty and fuzzy sets in objective things is described in more detail by using this new method. Interval-valued intuitionistic fuzzy set is also applied to various areas, for example, characteristic decision [17], logistic programming [18], and pattern recognition [19].

Therefore, in order to improve exact recognition ratios for aerial targets and avoid the "combination explosion" problem caused by intuitionistic fuzzy inference, this paper proposes a new method based on the interval-valued intuitionistic fuzzy sets with grey correlation.

Then the rest of paper is set out as follows. In Section 2, target recognition problem is described, and limitations of known approaches to target recognition are analyzed. In Section 3, a novel algorithm based on interval-valued intuitionistic fuzzy sets with grey correlation is proposed, which is free of these limitations. Recognition matrix of an aerial target is established first, and then grey correlation theory is used to analyze the recognition matrix. In Section 4, the proposed algorithm is adopted in different target recognition experimentally and compared with other previous algorithms. Finally, the concluding section summarizes the paper.

\section{Description of Target Recognition}

Assuming that there are $n$ categories of recognized targets for each of which $k$ feature parameters are gained by using multiple sensors. All $k$ feature parameters would be combined to form a feature vector that describes the target. In this paper, $\mathbf{U}_{1}=\left\{u_{1}, u_{2}, \ldots, u_{n}\right\}$ and $\mathbf{U}_{2}=\left\{a_{1}, a_{2}, \ldots, a_{k}\right\}$ are used to represent the target category set and target feature parameter set, respectively. Via measuring unknown target $\mathbf{X}$, the measurements of $\mathbf{X}=\left\{x_{1}, x_{2}, \ldots, x_{k}\right\}$ can be obtained. The so-called target recognition is actually that the feature parameter vector formed by measurements can be clustered to the most associated similar target category. Hence, the target recognition issue can be seen as a decision issue. By computing and analyzing the feature parameter $\mathbf{X}$, the most matching target category $u_{i}$ is directly chosen from target category set as the decisive result.

Note that the targets investigated in this paper are aerial targets. In order to make recognizing targets typicality and to enhance the recognition efficiency, target categories are, respectively, bomber $\left(\mathrm{B}\right.$, as $\left.u_{1}\right)$, gunship $\left(\mathrm{G}\right.$, as $\left.u_{2}\right)$, tactical ballistic missile (TBM, as $u_{3}$ ), cruise missile (CM, as $u_{4}$ ), and air-to-ground guided missile (AGM, as $u_{5}$ ). In these five target categories, the bomber has a large volume of missiles and significantly high attacking capability, while gunship is of low penetration and speed even if with high attacking capability. The tactical ballistic missile can have a very fast speed and should be intercepted in a very short time. Also, the cruise missile has a low speed during the time of cruise, while the air-to-ground guided missile can be significantly fast with a small radar cross section area.

As every target selected in this paper has its own prominent characteristics, the target feature parameter set can be set as follows:

$$
\mathbf{U}_{2}=\left\{S, H, V_{H}, V_{V}, A\right\},
$$

where $S$ represents the radiation cross section area of radar, $H$ is the height of target, $V_{H}$ indicates the cruise speed, $V_{V}$ indicates the vertical speed, and $A$ represents the acceleration of target.

Recognizing an aerial target means the access to the feature parameters such as radiation cross section area, height of target, speed, and acceleration from sensors, and via the analyses and comparisons of these parameters, the membership of this target to each category is identified, resulting in suggesting the specific category the target should belong to.

Grey correlation analysis method is applied to solve the target recognition problems. Guan and He [7] use this method to solve the radar emitter recognition problems, and Lin et al. [8] solve the radiation source recognition problems based on its improved method.

The observing vector of one unknown target in terms of each characteristic parameter can be denoted by $X_{0}=$ $\left\{X_{0}(j) \mid j=1,2, \ldots, 5\right\}$. Let $M$ data sets that are known in the target recognition database be $X_{m}=\left\{X_{m}(j) \mid j=\right.$ $1,2, \ldots, 5\}(m=1,2, \ldots, M)$. Using grey correlation method to recognize target, the grey correlation degree between unknown target $X_{0}$ and each known data $X_{m}$ should be calculated as follows:

$$
\begin{aligned}
& \xi_{m}(j) \\
& =\frac{\min _{m} \min _{j}\left|X_{0}(j)-X_{m}(j)\right|+\rho \max _{m} \max _{j}\left|X_{0}(j)-X_{m}(j)\right|}{\left|X_{0}(j)-X_{m}(j)\right|+\rho \max _{m} \max _{j}\left|X_{0}(j)-X_{m}(j)\right|},
\end{aligned}
$$

where $\rho$ is the resolution coefficient.

Then the known data with highest grey correlation degree can be correlated with the unknown target $X_{0}$.

This approach using grey correlation is simple and effective to resolve the issue of some default characteristic parameters. This method, however, needs a lot of data in the target recognition database, such that the final recognition results heavily depend on the existing knowledge. Therefore, if some data in the database is not accurate enough, the recognition results would be incorrect.

Another common method applied in target recognition problems is intuitionistic fuzzy inference, which is proposed by Lei et al. [14]. They first applies IFS to describe each characteristic parameter. The membership function and nonmembership function are chosen, based on which the inference rules can be established. Then, combining all inference rules 
terminally yields the target recognition results. The inference rules are established as follows:

$$
\text { If }, \ldots \text {, and }, \ldots \text {, Then }, \ldots \text {. }
$$

Using the membership function and nonmembership function, IFS can describe uncertain information quite well. And the credible inference rules can be used to recognize target accurately. Thus more credible inference rules lead to more accurate recognition results. However, large numbers of categories of target characteristic parameters and intuition fuzzy sets result in the multiple increasing on the fuzzy inference rules, such that the issue of "combinatorial explosion" correspondingly comes out. Moreover, in practice, the membership or nonmembership may not be denoted by real values, which is a constraint of the proposed method. For solving this drawback, the interval-valued intuitionistic fuzzy sets can be applied to describe the uncertain information.

Therefore, in order to combine the advantages of these two methods while improving their drawbacks, a novel algorithm or method should be proposed. The new method should describe uncertain feature and uncertain information just like the IFS method. Furthermore, interval-valued intuitionistic fuzzy sets can describe uncertain information better. On the other hand, the new method should have the ability to analyze uncertain information as grey correlation analysis method. So the algorithm for target recognition based on interval-valued intuitionistic fuzzy sets with grey correlation is proposed.

\section{Algorithm of Interval-Valued Intuitionistic Fuzzy Sets with Grey Correlation}

This section presents an algorithm for target recognition by combining interval-valued intuitionistic fuzzy sets with grey correlation. When using the proposed algorithm to recognize an unknown target, one should first establish a target recognition matrix composed of interval-valued intuitionistic fuzzy numbers. Each interval-valued intuitionistic fuzzy number represents the interval-valued membership and nonmembership of the unknown target to one category in terms of one characteristic parameter. In order to realize the recognition of this target, grey correlation method is used to analyze the target recognition matrix. Then grey correlation sequence is subsequently obtained. Figure 1 shows the flow chart of the proposed algorithm.

3.1. Interval-Valued Intuitionistic Fuzzy Numbers. Assuming that $\mathbf{X}$ is a constant domain, we say that

$$
\mathbf{A}=\left\{\left\langle x, \mu_{A}(x), \gamma_{A}(x)\right\rangle \mid x \in \mathbf{X}\right\}
$$

is an intuitionistic fuzzy set on $\mathbf{X}$. In this set, $\mu_{A}: \mathbf{X} \rightarrow[0,1]$ and $\gamma_{A}: \mathbf{X} \rightarrow[0,1]$, respectively, represent the membership and nonmembership functions from $x$ to $\mathbf{A}$. Moreover, for all $x \in \mathbf{X}$ in $\mathbf{A}, 0 \leqslant \mu_{A}(x)+\gamma_{A}(x) \leqslant 1$ holds true.

Due to the uncertainty of objective things, it is really difficult that $\mu_{A}(x)$ and $\gamma_{A}(x)$ can be expressed by exact real values. Therefore the interval values are introduced, which

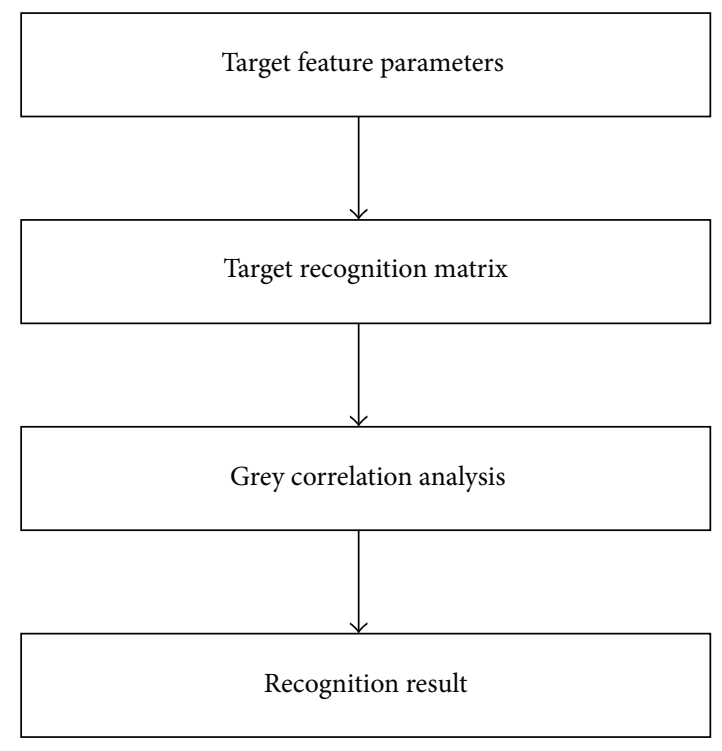

FIGURE 1: Flowchart of algorithm for target recognition.

means that $\mu_{A}(x)$ and $\gamma_{A}(x)$ are given by the interval values in the interval of $[0,1]$. Furthermore, for any $x \in \mathbf{X}, 0 \leqslant$ $\sup \mu_{A}(x)+\sup \gamma_{A}(x) \leqslant 1$ holds true.

Therefore, the sequential intervals formed by membership and nonmembership intervals are called interval-valued intuitionistic fuzzy number, which is $([a, b],[c, d])$, where $[a, b] \subset[0,1]$ and $[c, d] \subset[0,1], b+d \leqslant 1$.

\subsection{Interval-Valued Intuitionistic Fuzzy Recognition Matrix.} The target recognition matrix of an unknown target is constructed as follows:

$$
\mathbf{R}=\left(\alpha_{i j}\right)_{n \times k} \quad(i=1,2, \ldots, n ; j=1,2, \ldots, k),
$$

where $\alpha_{i j}$ is an interval-valued intuitionistic fuzzy number, which is composed of the interval-valued membership and the interval-valued nonmembership of the unknown target to category $u_{i}$ in terms of parameter $a_{j}$. It is shown that

$$
\alpha_{i j}=\left(\left[\inf \mu_{i j}, \sup \mu_{i j}\right],\left[\inf \gamma_{i j}, \sup \gamma_{i j}\right]\right),
$$

where inf $\mu_{i j}$ and $\sup \mu_{i j}$, respectively, represent the lower bound and upper bound of membership interval of an unknown target to category $u_{i}$ in terms of parameter $a_{j}$ and inf $\gamma_{i j}$ and $\sup \gamma_{i j}$, respectively, represent the lower bound and upper bound of nonmembership interval of the target to category $u_{i}$ in terms of parameter $a_{j}$.

For instance, $\alpha_{i j}=([0.6,0.8],[0.1,0.2])$ indicates via the feature parameter $a_{j}$ the likelihood of recognizing an unknown target as $u_{i}$ is between $60 \%$ and $80 \%$, while the likelihood that the target does not belong to $u_{i}$ is between $10 \%$ and $20 \%$.

The selection of upper and lower bounds is a significantly key part for the construction of target matrix. For example, $\mu_{\mathrm{TBM}}\left(V_{H}\right)$ represents the membership of an unknown target to TBM in terms of the cruise speed $V_{H}$, and $\gamma_{\mathrm{TBM}}\left(V_{H}\right)$ represents the nonmembership of the unknown target to TBM 
in terms of the cruise speed $V_{H}$. Let $V_{H}$ lie in $[0,3000 \mathrm{~m} / \mathrm{s}]$ as the speed of TBM is very fast, which is typically 4-6 times the speed of sound. By considering the nonlinearity of membership and nonmembership functions, the piecewise functions are applied to solve the upper bound and lower bound of $\mu_{\mathrm{TBM}}\left(V_{H}\right)$ and $\gamma_{\mathrm{TBM}}\left(V_{H}\right)$ as follows:

$$
\begin{aligned}
& \inf \mu_{\mathrm{TBM}}\left(V_{H}\right) \\
& = \begin{cases}\frac{V_{H}}{5000}, & V_{H} \leqslant 1000 \mathrm{~m} / \mathrm{s} \\
\frac{\left(V_{H}-1000\right)}{1000}+0.2, & 1000 \mathrm{~m} / \mathrm{s}<V_{H} \leqslant 1400 \mathrm{~m} / \mathrm{s} \\
\frac{\left(V_{H}-1400\right)}{1500}+0.6, & 1400 \mathrm{~m} / \mathrm{s}<V_{H} \leqslant 1700 \mathrm{~m} / \mathrm{s} \\
\frac{\left(1700-V_{H}\right)}{1000}+0.8, & 1700 \mathrm{~m} / \mathrm{s}<V_{H} \leqslant 2000 \mathrm{~m} / \mathrm{s} \\
\frac{\left(2000-V_{H}\right)}{2500}+0.5, & 2000 \mathrm{~m} / \mathrm{s}<V_{H} \leqslant 3000 \mathrm{~m} / \mathrm{s},\end{cases}
\end{aligned}
$$$$
\sup \mu_{\mathrm{TBM}}\left(V_{H}\right)
$$$$
= \begin{cases}\frac{V_{H}}{10000}+0.2, & V_{H} \leqslant 1000 \mathrm{~m} / \mathrm{s} \\ \frac{\left(V_{H}-1000\right)}{1000}+0.3, & 1000 \mathrm{~m} / \mathrm{s}<V_{H} \leqslant 1400 \mathrm{~m} / \mathrm{s} \\ \frac{\left(V_{H}-1400\right)}{1500}+0.7, & 1400 \mathrm{~m} / \mathrm{s}<V_{H} \leqslant 1700 \mathrm{~m} / \mathrm{s} \\ \frac{\left(1700-V_{H}\right)}{1000}+0.9, & 1700 \mathrm{~m} / \mathrm{s}<V_{H} \leqslant 2000 \mathrm{~m} / \mathrm{s} \\ \frac{\left(2000-V_{H}\right)}{2500}+0.6, & 2000 \mathrm{~m} / \mathrm{s}<V_{H} \leqslant 3000 \mathrm{~m} / \mathrm{s},\end{cases}
$$$$
\inf \gamma_{\mathrm{TBM}}\left(V_{H}\right)
$$$$
= \begin{cases}-\frac{V_{H}}{10000}+0.7, & V_{H} \leqslant 1000 \mathrm{~m} / \mathrm{s} \\ \frac{\left(1000-V_{H}\right)}{1000}+0.6, & 1000 \mathrm{~m} / \mathrm{s}<V_{H} \leqslant 1400 \mathrm{~m} / \mathrm{s} \\ \frac{\left(1400-V_{H}\right)}{1500}+0.2, & 1400 \mathrm{~m} / \mathrm{s}<V_{H} \leqslant 1700 \mathrm{~m} / \mathrm{s} \\ \frac{\left(V_{H}-1700\right)}{1000}, & 1700 \mathrm{~m} / \mathrm{s}<V_{H} \leqslant 2000 \mathrm{~m} / \mathrm{s} \\ \frac{\left(V_{H}-2000\right)}{2500}+0.3, & 2000 \mathrm{~m} / \mathrm{s}<V_{H} \leqslant 3000 \mathrm{~m} / \mathrm{s},\end{cases}
$$

$\sup \gamma_{\text {ТВM }}\left(V_{H}\right)$

$$
= \begin{cases}-\frac{V_{H}}{5000}+0.9, & V_{H} \leqslant 1000 \mathrm{~m} / \mathrm{s} \\ \frac{\left(1000-V_{H}\right)}{1000}+0.7, & 1000 \mathrm{~m} / \mathrm{s}<V_{H} \leqslant 1400 \mathrm{~m} / \mathrm{s} \\ \frac{\left(1400-V_{H}\right)}{1500}+0.3, & 1400 \mathrm{~m} / \mathrm{s}<V_{H} \leqslant 1700 \mathrm{~m} / \mathrm{s} \\ \frac{\left(V_{H}-1700\right)}{1000}+0.1, & 1700 \mathrm{~m} / \mathrm{s}<V_{H} \leqslant 2000 \mathrm{~m} / \mathrm{s} \\ \frac{\left(V_{H}-2000\right)}{2500}+0.4, & 2000 \mathrm{~m} / \mathrm{s}<V_{H} \leqslant 3000 \mathrm{~m} / \mathrm{s} .\end{cases}
$$

When the cruise speed of one aerial target is known, based on it, the membership and nonmembership interval can be calculated according to (7). For example, when $V_{H}=$ $2000 \mathrm{~m} / \mathrm{s},(8)$ is obtained as follows:

$$
\alpha_{\mathrm{TBM}_{-} V_{H}}=([0.5,0.6],[0.3,0.4]) .
$$

Therefore, when the cruise speed is $2000 \mathrm{~m} / \mathrm{s}$, according to (8), the likelihood of recognizing this target as TBM via $V_{H}$ is between $50 \%$ and $60 \%$, while the impossibility is between $30 \%$ and $40 \%$.

Besides that, the TBM has the significant characteristics of small radiation cross section area, high vertical speed, and large height and acceleration. According to those characteristics, the upper and lower bounds of memberships and nonmemberships of $\alpha_{\mathrm{TBM}-S}, \alpha_{\mathrm{TBM}-H}, \alpha_{\mathrm{TBM}-V_{V}}$ and $\alpha_{\mathrm{TBM}-A}$ can be found typically.

Also, the piecewise functions are applied to establish the upper bound and lower bound of $\mu_{\mathrm{AGM}}(H)$ and $\gamma_{\mathrm{AGM}}(H)$ as

$$
\begin{aligned}
& \inf \mu_{\mathrm{AGM}}(H) \\
& = \begin{cases}0 & H \leqslant 3000 \mathrm{~m} \\
\frac{(H-3000)}{10000} & 3000 \mathrm{~m}<H \leqslant 11000 \mathrm{~m} \\
\frac{(11000-H)}{30000}+0.8 & 11000 \mathrm{~m}<H \leqslant 17000 \mathrm{~m} \\
\frac{(17000-H)}{60000}+0.6 & H \geqslant 17000 \mathrm{~m},\end{cases}
\end{aligned}
$$

$\sup \mu_{\mathrm{AGM}}(H)$

$$
= \begin{cases}0.1 & H \leqslant 3000 \mathrm{~m} \\ \frac{(H-3000)}{10000}+0.1 & 3000 \mathrm{~m}<H \leqslant 11000 \mathrm{~m} \\ \frac{(11000-H)}{30000}+0.9 & 11000 \mathrm{~m}<H \leqslant 17000 \mathrm{~m} \\ \frac{(17000-H)}{60000}+0.7 & H \geqslant 17000 \mathrm{~m},\end{cases}
$$

$\inf \gamma_{\text {AGM }}(H)$

$$
= \begin{cases}0.8 & H \leqslant 3000 \mathrm{~m} \\ \frac{(3000-H)}{10000}+0.8 & 3000 \mathrm{~m}<H \leqslant 11000 \mathrm{~m} \\ \frac{(H-11000)}{40000} & 11000 \mathrm{~m}<H \leqslant 17000 \mathrm{~m} \\ \frac{(H-17000)}{60000}+0.15 & H>17000 \mathrm{~m},\end{cases}
$$

$\sup \gamma_{\mathrm{AGM}}(H)$

$$
= \begin{cases}0.9 & H \leqslant 3000 \mathrm{~m} \\ \frac{(3000-H)}{10000}+0.9 & 3000 \mathrm{~m}<H \leqslant 11000 \mathrm{~m} \\ \frac{(H-11000)}{40000}+0.1 & 11000 \mathrm{~m}<H \leqslant 17000 \mathrm{~m} \\ \frac{(H-17000)}{60000}+0.25 & H>17000 \mathrm{~m} .\end{cases}
$$

Similarly, the memberships and nonmemberships of other categories can be established by the same method. 
This section would not present the details due to the paper space. Hence, all of the obtained interval-valued intuitionistic fuzzy numbers form the target recognition matrices of the unknown target.

3.3. Grey Correlation. In this part, grey correlation theory is used to analyze the recognition matrix established above.

First, positive desired recognition strategy and negative desired recognition strategy of an unknown target are, respectively, built up. The positive desired recognition strategy is composed of the maximal number of each column of the recognition matrix, and the negative desired recognition strategy is composed of the minimal number of every column of the recognition matrix. So the score function and accuracy function are used to compare the interval-valued intuitionistic fuzzy numbers.

Second, grey correlation coefficient matrix of the unknown target is built up. Each grey correlation coefficient is the relationship between each entry in the recognition matrix and each entry of desired recognition strategy. According to grey correlation analysis method, the grey correlation coefficient of every entry of the recognition matrix is calculated.

Finally, grey correlation sequence of the unknown target is built up. Grey correlation sequence is composed of grey correlation degrees. Via the grey correlation coefficient matrix, the grey correlation degree of the unknown target to each category is calculated. The category which has the maximal grey correlation degree should be selected as the optimal recognition category.

\subsubsection{Desired Recognition Strategy}

Definition 1 (score function and accuracy function). Let $\alpha=([a, b],[c, d])$ be an interval-valued intuitionistic fuzzy number. Its score function is defined as $\Delta(\alpha)=(a-c+b-d) / 2$, while the so-called accuracy function is $H(\alpha)=(a+c+b+$ d) $/ 2$.

Definition 2. Assume that $\alpha_{1}$ and $\alpha_{2}$ are two interval-valued intuitionistic fuzzy numbers. If $\Delta\left(\alpha_{1}\right)<\Delta\left(\alpha_{2}\right)$, then $\alpha_{1}<\alpha_{2}$. In particular, when $\Delta\left(\alpha_{1}\right)=\Delta\left(\alpha_{2}\right)$, if $H\left(\alpha_{1}\right)<H\left(\alpha_{2}\right)$, then $\alpha_{1}<\alpha_{2}$ holds.

Definition 3 (positive and negative desired recognition strategies). The interval-valued intuitionistic fuzzy vectors are as follows:

$$
\begin{aligned}
& x^{+}=\left\{\alpha_{1}^{+}, \alpha_{2}^{+}, \ldots, \alpha_{k}^{+}\right\}, \\
& x^{-}=\left\{\alpha_{1}^{-}, \alpha_{2}^{-}, \ldots, \alpha_{k}^{-}\right\},
\end{aligned}
$$

which are, respectively, positive and negative desired recognition strategies of the target recognition matrix $\left(\alpha_{i j}\right)_{n \times k}$, if and only if

$$
\begin{array}{ll}
\alpha_{j}^{+}=\max _{i}\left\{\alpha_{i j}\right\} & (j=1,2, \ldots, k), \\
\alpha_{j}^{-}=\min _{i}\left\{\alpha_{i j}\right\} \quad(j=1,2, \ldots, k)
\end{array}
$$

hold true.
From the definitions above, the scales of two intervalvalued intuitionistic fuzzy numbers can be determined directly by the score and accuracy functions. It is typical that the associated fuzzy number would be larger with the larger score function, while if the score functions of two fuzzy numbers are equal, then the larger fuzzy sets are is only dependent on the larger accuracy function.

Based on the determined rules described above, to solve the positive or negative desired strategy is necessarily to extract the maximal or minimal interval-valued intuitionistic fuzzy number in each column from the target recognition matrix.

3.3.2. Grey Correlation Coefficient Matrix. After obtaining the positive and negative desired recognition strategies, this part calculates the grey correlation coefficient between each entry in the target recognition matrix and each entry of the strategy.

Each grey correlation coefficient represents the proximity between each entry in the target recognition matrix and each entry of the strategy. The closer $\alpha_{i j}$ and $\alpha_{j}^{+}$are, the farther $\alpha_{i j}$ and $\alpha_{j}^{-}$are, and the better will be.

As $\left|X_{0}(j)-X_{m}(j)\right|$ in (2), the proximity between $\alpha_{i j}$ and $\alpha_{j}^{+}\left(\alpha_{j}^{-}\right)$should be calculated first. Let $D_{i j}^{+}\left(D_{i j}^{-}\right)$represent the distance. Use the norms of interval values to calculate the distance as follows:

$$
\begin{aligned}
D_{i j}^{+} & =D_{i j}^{+}\left(\alpha_{i j}, \alpha_{j}^{+}\right)=\frac{1}{4}\left(\left|\inf v-\inf \mu_{j}^{+}\right|\right. \\
& +\left|\sup \mu_{i j}-\sup \mu_{j}^{+}\right|+\left|\inf \gamma_{i j}-\inf \gamma_{j}^{+}\right| \\
& \left.+\left|\sup \gamma_{i j}-\sup \gamma_{j}^{+}\right|\right), \\
D_{i j}^{-} & =D_{i j}^{-}\left(\alpha_{i j}, \alpha_{j}^{-}\right)=\frac{1}{4}\left(\left|\inf \mu_{i j}-\inf \mu_{j}^{-}\right|\right. \\
& +\left|\sup \mu_{i j}-\sup \mu_{j}^{-}\right|+\left|\inf \gamma_{i j}-\inf \gamma_{j}^{-}\right| \\
& \left.+\left|\sup \gamma_{i j}-\sup \gamma_{j}^{-}\right|\right) .
\end{aligned}
$$

Then distance matrix $\left(D_{i j}^{+}\right)_{n \times k}$ consists of all of the distance $D_{i j}^{+}$between $\alpha_{i j}$ and $\alpha_{j}^{+}$. $\left(D_{i j}^{-}\right)_{n \times k}$ consists of all of the distance $D_{i j}^{-}$between $\alpha_{i j}$ and $\alpha_{j}^{-}$.

And then, $\xi_{i j}^{+}$is defined as grey correlation coefficient between $\alpha_{i j}$ and $\alpha_{j}^{+}$. And $\xi_{i j}^{-}$is coefficient between $\alpha_{i j}$ and $\alpha_{j}^{-}$. Using grey system theory and the distance matrices, the formula of grey correlation coefficients can be obtained as follows:

$$
\begin{aligned}
& \xi_{i j}^{+}=\frac{\min _{i, j} D_{i j}^{+}+\rho \max _{i, j} D_{i j}^{+}}{D_{i j}^{+}+\rho \max _{i, j} D_{i j}^{+}}, \\
& \xi_{i j}^{-}=\frac{\min _{i, j} D_{i j}^{-}+\rho \max _{i, j} D_{i j}^{-}}{D_{i j}^{-}+\rho \max _{i, j} D_{i j}^{-}},
\end{aligned}
$$

where $\rho$ is the recognition coefficient that is typically 0.5 . 
In (13), $\max _{i, j} D_{i j}^{+}$and $\min _{i, j} D_{i j}^{+}$, respectively, represent the maximal and minimal element of the distance matrix $\left(D_{i j}^{+}\right)_{n \times k}$. In (14), $\max _{i, j} D_{i j}^{-}$and $\min _{i, j} D_{i j}^{-}$, respectively, represent the maximal and minimal element of the distance matrix $\left(D_{i j}^{-}\right)_{n \times k}$.

All of grey correlation coefficients would form the grey correlation coefficient matrices; namely, that are $\left(\xi_{i j}^{+}\right)_{n \times k}$ and $\left(\xi_{i j}^{-}\right)_{n \times k}$.

3.3.3. Grey Correlation Sequence. In this part, the grey correlation degree between each target category $u_{i}$ and the positive or the negative desired strategy is calculated. By using the grey correlation coefficient matrices, the grey correlation degrees, $\lambda_{i}^{+}$and $\lambda_{i}^{-}$, are calculated, respectively, as follows:

$$
\begin{aligned}
& \lambda_{i}^{+}=\sum_{j=1}^{k} \xi_{i j}^{+} a_{j}, \\
& \lambda_{i}^{-}=\sum_{j=1}^{k} \xi_{i j}^{-} a_{j},
\end{aligned}
$$

where $a_{j}$ represents the weight of the feature parameter, and it follows the properties of $a_{j} \geqslant 0$ and $\sum_{j=1}^{k} a_{j}=1$.

The special case is that $a_{j}$ is $1 / k$, when the importance of each feature parameter is treated to be equal, which is naturally the averaged case. In practice, the selection of weights, however, enables the sum of errors to be minimal between the grey correlation degree of each target category and the grey correlation degree of desired recognition strategy, namely, which follows the optimization model:

$$
\begin{array}{ll}
\min & F(a)=\sum_{i=1}^{n} \sum_{j=1}^{k}\left[\left(1-\xi_{i j}^{+}\right) a_{j}\right]^{2}, \\
\text { s.t. } & \sum_{j=1}^{k} a_{j}=1 \\
& a_{j} \geqslant 0 .
\end{array}
$$

By solving this model, we have

$$
a_{j}=\frac{\left\{\sum_{j=1}^{k}\left[\sum_{i=1}^{n}\left(1-\xi_{i j}^{+}\right)^{2}\right]^{-1}\right\}^{-1}}{\sum_{i=1}^{n}\left(1-\xi_{i j}^{+}\right)^{2}} \text {. }
$$

Via (15) (17), the grey correlation degree of each target category can be obtained. While $\lambda_{i}^{+}$becomes larger and $\lambda_{i}^{-}$ becomes smaller, the target category $u_{i}$ is closer to the positive desired recognition strategy and more keeping away from the negative desired recognition strategy.

Introduce another definition associated with comprehensive grey correlation degree as follows:

$$
d_{i}=\frac{\left(\lambda_{i}^{+}\right)^{2}}{\left(\lambda_{i}^{+}\right)^{2}+\left(\lambda_{i}^{-}\right)^{2}},
$$

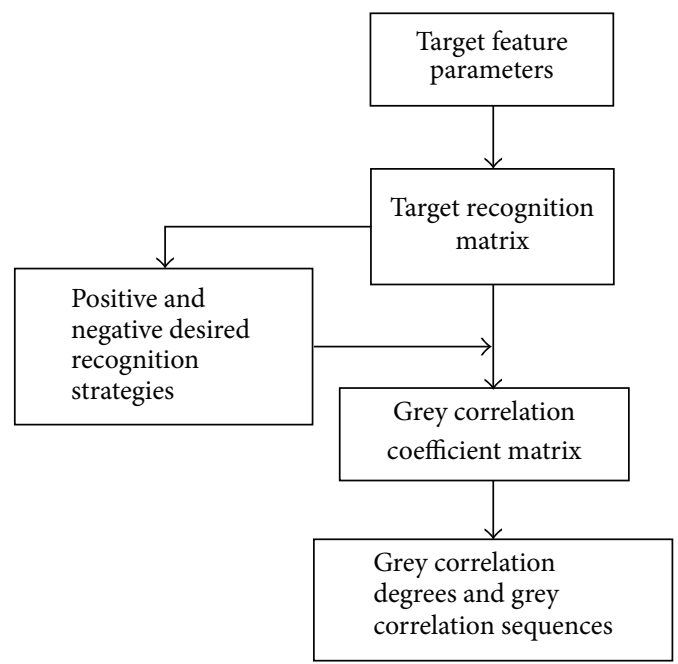

FIGURE 2: Flowchart of software for target recognition.

which is typically used to represent the level that $u_{i}$ belongs to the positive and negative desired recognition strategies. This comprehensive grey correlation degree also indicates that the target category $u_{i}$ belongs to the positive desired recognition strategy with the level of $d_{i}$, while belonging to the negative desired recognition strategy with the level of $1-d_{i}$. It is furthermore seen that the confidence increases that the target is recognized as $u_{i}$, when $d_{i}$ increases.

Then the grey correlation sequence of the unknown target can be obtained by descending the comprehensive grey correlation degrees. According to the maximal correlation recognition, the category which has the maximal comprehensive grey correlation would be recognized as the target category associated with the unknown target.

\section{Application of the Proposed Algorithm}

4.1. Target Recognition Processing Software. Based on the proposed algorithm presented before, a target recognition processing software is established by plugging the membership and nonmembership functions into the software. Then defining the feature parameters of targets as inputs the target recognition matrices, positive and negative desired recognition strategies, grey correlation coefficient matrices, and grey correlation degrees can be subsequently calculated. And after that the final output is the grey correlation sequence of each target category. The final recognition strategy is the maximal grey correlation sequence that is the tool to determine the target category. Figure 2 shows the flow chart of the proposed software.

In order to validate the efficacy and stability of the proposed algorithm this paper uses 200 sets of feature parameters selected randomly according to the uniform distribution from the database as inputs to testify the processing capability of the software. These 200 sets of feature parameters correspond, respectively, to the proposed 5 target categories, which means every 40 sets of data would be for each target category. Part of measured raw data is shown in Table 1. 
Consider the 1st feature parameter vector as an example in Table 1 as follows:

$$
\{0.5,35000,1800,2000,350\}
$$

which represents that the radiation cross section area of this target is $0.5 \mathrm{~m}^{2}$; the height of the target is $35000 \mathrm{~m}$; its cruise speed is $1800 \mathrm{~m} / \mathrm{s}$; its vertical speed is $2000 \mathrm{~m} / \mathrm{s}$; and acceleration is $350 \mathrm{~m} / \mathrm{s}^{2}$. This section uses this vector as an example to illustrate the algorithm process of the target recognition processing software.

(1) Target Recognition Matrix. Using the mentioned membership and nonmembership interval-valued intuitionistic fuzzy functions, the target recognition matrix of relevance is constructed as follows:

$\mathbf{R}$

$$
=\left[\begin{array}{ccccc}
([0.05,0.15],[0.6,0.7]) & ([0.35,0.45],[0.4,0.5]) & ([0.11,0.21],[0.69,0.79]) & ([0.1,0.2],[0.7,0.8]) & ([0.1,0.2],[0.7,0.8]) \\
([0,0.1],[0.65,0.75]) & ([0.1,0.2],[0.7,0.8]) & ([0.05,0.15],[0.7,0.8]) & ([0.02,0.12],[0.78,0.88]) & ([0.1,0.2],[0.65,0.75]) \\
([0.55,0.65],[0.1,0.2]) & ([0.7,0.8],[0.1,0.2]) & ([0.7,0.8],[0.1,0.2]) & ([0.7,0.8],[0.1,0.2]) & ([0.7,0.8],[0.1,0.2]) \\
([0.65,0.75],[0,0.1]) & ([0,0.1],[0.7,0.8]) & ([0.17,0.27],[0.58,0.68]) & ([0,0.1],[0.75,0.85]) & ([0,0.1],[0.7,0.8]) \\
([0.6,0.7],[0.05,0.15]) & ([0.3,0.4],[0.45,0.55]) & ([0.5,0.6],[0.3,0.4]) & ([0.6,0.7],[0.2,0.3]) & ([0.8,0.9],[0,0.1])
\end{array}\right] .
$$

(2) Desired Recognition Strategies. $([0.65,0.75],[0,0.1])$ is the maximal entries of the first column of the target recognition matrix. And $([0.7,0.8],[0.1,0.2]),([0.7,0.8],[0.1,0.2])$, $([0.7,0.8],[0.1,0.2])$, and $([0.8,0.9],[0.0 .1])$ are, respectively, the maximal entries of other four columns.

$([0,0.1],[0.65,0.75])$ is the minimal entries of the first column of the target recognition matrix. And $([0,0.1],[0.7,0.8]), \quad([0.05,0.15],[0.7,0.8]), \quad([0.02,0.12]$, $[0.78,0.88])$, and $([0,0.1],[0.7,0.8])$ are, respectively, the minimal entries of other four columns.

Therefore the positive and negative desired recognition strategies can be gained by adopting score function and accuracy function as follows:

$$
\begin{aligned}
x^{+} & =\{([0.65,0.75],[0,0.1]),([0.7,0.8],[0.1,0.2]), \\
& ([0.7,0.8],[0.1,0.2]),([0.7,0.8],[0.1,0.2]), \\
& ([0.8,0.9],[0,0.1])\}, \\
x^{-} & =\{([0,0.1],[0.65,0.75]),([0,0.1],[0.7,0.8]), \\
& ([0.05,0.15],[0.7,0.8]),([0.02,0.12],[0.78,0.88]), \\
& ([0,0.1],[0.7,0.8])\} .
\end{aligned}
$$

(3) Grey Correlation Coefficient Matrix. Then (12) are applied to compute the distance matrices:

$$
\begin{aligned}
\mathbf{D}^{+} & =\left(D_{i j}^{+}\right)_{n \times k} \\
& =\left[\begin{array}{ccccc}
0.6 & 0.325 & 0.59 & 0.6 & 0.7 \\
0.65 & 0.6 & 0.625 & 0.68 & 0.675 \\
0.1 & 0 & 0 & 0 & 0.1 \\
0 & 0.65 & 0.505 & 0.675 & 0.7 \\
0.05 & 0.375 & 0.2 & 0.1 & 0
\end{array}\right],
\end{aligned}
$$

$$
\begin{aligned}
\mathbf{D}^{-} & =\left(D_{i j}^{-}\right)_{n \times k} \\
& =\left[\begin{array}{ccccc}
0.05 & 0.325 & 0.035 & 0.08 & 0.05 \\
0 & 0.05 & 0 & 0 & 0.075 \\
0.55 & 0.65 & 0.625 & 0.68 & 0.65 \\
0.65 & 0 & 0.12 & 0.025 & 0 \\
0.6 & 0.275 & 0.425 & 0.58 & 0.75
\end{array}\right] .
\end{aligned}
$$

From (22), the maximal entry of the distance matrix $\mathbf{D}^{+}$ is 0.7 , and the minimal entry is 0 . So $\max _{i, j} D_{i j}^{+}=0.7$ and $\min _{i, j} D_{i j}^{+}=0$. On the other hand, $\max _{i, j} D_{i j}^{-}=0.75$ and $\min _{i, j} D_{i j}^{-}=0$.

The distance matrices and grey correlation coefficients yield the grey correlation coefficient matrices of the target as follows:

$$
\begin{aligned}
& \xi^{+}=\left(\xi_{i j}^{+}\right)_{n \times k} \\
& =\left[\begin{array}{ccccc}
0.3684 & 0.5185 & 0.3723 & 0.3684 & 0.3333 \\
0.35 & 0.3684 & 0.3590 & 0.3398 & 0.3415 \\
0.7778 & 1 & 1 & 1 & 0.7778 \\
1 & 0.35 & 0.4094 & 0.3415 & 0.3333 \\
0.875 & 0.4828 & 0.6364 & 0.7778 & 1
\end{array}\right], \\
& \boldsymbol{\xi}^{-}=\left(\xi_{i j}^{-}\right)_{n \times k} \\
& =\left[\begin{array}{ccccc}
0.8824 & 0.5357 & 0.9146 & 0.8242 & 0.8824 \\
1 & 0.8824 & 1 & 1 & 0.8333 \\
0.4054 & 0.3659 & 0.375 & 0.3555 & 0.3659 \\
0.3659 & 1 & 0.7576 & 0.9375 & 1 \\
0.3846 & 0.5769 & 0.4688 & 0.3927 & 0.3333
\end{array}\right] .
\end{aligned}
$$


TABLE 1: Part of original data.

\begin{tabular}{ccccccc}
\hline & $S\left(\mathrm{~m}^{2}\right)$ & $H(\mathrm{~m})$ & $V_{V}(\mathrm{~m} / \mathrm{s})$ & $V_{H}(\mathrm{~m} / \mathrm{s})$ & $A\left(\mathrm{~m} / \mathrm{s}^{2}\right)$ & $\begin{array}{c}\text { Recognition } \\
\text { result }\end{array}$ \\
\hline 1 & 0.5 & 35000 & 1800 & 2000 & 350 & $u_{3}$ \\
2 & 0.6 & 40000 & 2000 & 1950 & 420 & $u_{3}$ \\
\hline 3 & 10 & 15000 & 250 & 8 & 0.5 & $u_{1}$ \\
4 & 15 & 12000 & 270 & 10 & 0.7 & $u_{1}$ \\
\hline 5 & 0.44 & 90 & 340 & 0 & 0.2 & $u_{4}$ \\
6 & 0.67 & 75 & 300 & 0 & 0.15 & $u_{4}$ \\
\hline 7 & 5 & 3000 & 70 & 10 & 0.5 & $u_{2}$ \\
8 & 3 & 2000 & 60 & 12 & 0.4 & $u_{2}$ \\
\hline 9 & 0.7 & 10000 & 1400 & 1500 & 400 & $u_{5}$ \\
10 & 0.8 & 11000 & 1500 & 1300 & 390 & $u_{5}$ \\
\hline
\end{tabular}

(4) Grey Correlation Sequence. By substituting the correlation coefficient matrix (23) into (17), the weights of feature parameters are represented by the following vector:

$$
\begin{aligned}
\mathbf{a} & =\left(a_{1}, a_{2}, a_{3}, a_{4}, a_{5}\right)^{T} \\
& =\left(\begin{array}{lllll}
0.2718 & 0.1824 & 0.1874 & 0.1828 & 0.1756
\end{array}\right)^{T} .
\end{aligned}
$$

By substituting (24) into (15), the grey correlation degree between each target category and the positive desired recognition strategy is

$$
\begin{aligned}
\lambda^{+} & =\left(\lambda_{i}^{+}\right)_{n \times 1} \\
& =\left(\begin{array}{llllll}
0.3903 & 0.3517 & 0.9006 & 0.5333 & 0.7629
\end{array}\right)^{T},
\end{aligned}
$$

and the grey correlation degree between each target category and the negative desired recognition strategy is

$$
\begin{aligned}
\lambda^{-} & =\left(\lambda_{i}^{-}\right)_{n \times 1} \\
& =\left(\begin{array}{llllll}
0.8146 & 0.9493 & 0.3764 & 0.7708 & 0.4279
\end{array}\right)^{T} .
\end{aligned}
$$

Using (18), the comprehensive correlation degree of each target category can be calculated as follows:

$$
\begin{aligned}
d & =\left(d_{i}\right)_{n \times 1} \\
& =\left(\begin{array}{llllll}
0.1867 & 0.1206 & 0.8513 & 0.3237 & 0.8180
\end{array}\right)^{T} .
\end{aligned}
$$

Hence, the sequence is $d_{3}>d_{5}>d_{4}>d_{1}>d_{2}$. The comprehensive correlation degree $d_{3}$ corresponding to the target category $u_{3}$ is maximal. It means that according to the five feature parameters the unknown target is closest to the feature parameters of TBM. It also demonstrates that the strategy of recognizing the unknown target as the TBM is the best.

The radiation reflection cross section area of this target is 1 to 2 magnitudes smaller than that of the airplane, while the cruise and vertical speeds are comparatively bigger. Moreover, the height is in a very high degree and acceleration is approximately 1 mach. Each feature parameter of
TABLE 2: Comparison of recognition results.

\begin{tabular}{lcc}
\hline Number & Algorithm & $\begin{array}{c}\text { Correct recognition } \\
\text { ratio (\%) }\end{array}$ \\
\hline Algorithm 1 & $\begin{array}{c}\text { Grey correlation } \\
\text { Intuitionistic fuzzy } \\
\text { Algorithm 2 }\end{array}$ & 92.5 \\
Algorithm 3 & $\begin{array}{c}\text { The proposed } \\
\text { algorithm }\end{array}$ & 96.5 \\
\hline
\end{tabular}

the unknown target matches quite well the feature parameters of TBM. The category of this target in the target recognition database is consistent with the recognition result by the interval-valued intuitionistic fuzzy sets with grey correlation algorithm. Such result also signifies that the proposed algorithm in this paper has good accuracy and high adaptation.

Table 1 lists each recognition result for each target. All of recognition results are consistent with the categories in the target recognition database. Therefore this proposed algorithm can be of stability in multiple target recognition problems.

4.2. Comparisons with Other Algorithms. Using the selected 200 data sets, the proposed algorithm in this paper is, respectively, compared to target recognition algorithms based on grey correlation [7] and intuitionistic fuzzy inference [14].

For intuitionistic fuzzy inference algorithm, the five parameters in (1) are similarly selected as the feature parameters. The numbers of membership functions are, respectively, $3,5,6,4$, and 4 . Therefore in this algorithm, the number of inference rules is up to $3 \times 5 \times 6 \times 4 \times 4=1440$.

Table 2 displays the experimental results by using three algorithms.

According to Table 2, for the target recognition issue, the proposed algorithm in this paper based on interval-valued intuitionistic fuzzy sets with grey correlation has a higher recognition ratio than that of algorithm 1 and algorithm 2 . This experiment also typically verifies that the algorithm is capable of dealing with the target recognition problems with uncertain information. The recognition results are closer to the practical cases.

Moreover, due to the numerous inference rules in algorithm 2, while the number of feature parameters increases, the number of inference rules will correspondingly increase in times. In such a case the issue of combination explosion easily comes up. However, for the proposed algorithm in this paper, only 25 membership and 25 nonmembership functions are needed. These functions are used for constructing a target recognition matrix, and the rest of algorithm processing is just element extractions and matrices computation for the target recognition matrix. Therefore, comparing with algorithm 2, algorithm 3 is more concise and simpler in algorithm construction and software compiling and as well can effectively avoid the issue of combination explosion.

According to the examples shown above, the novel method can describe the uncertain feature or uncertain information quite well. On the other hand, it has a good ability to analyze the uncertain information. Therefore the proposed 
method can be used to deal with the uncertain inference problems, such as the target recognition problems, the threat assessment problems, and the decision making problems. It can construct a relation between the feature parameters and the decision results based on the interval-valued intuitionistic fuzzy sets with grey correlation method.

\section{Conclusion}

The paper introduces a target recognition algorithm based on interval-valued intuitionistic fuzzy sets with grey correlation. Using interval-valued intuitionistic fuzzy numbers, the target recognition matrix of an unknown target is established, from which the positive and negative desired recognition strategies are extracted. According to grey system theory, the grey correlation degree between the unknown target and each category is built up to obtain the optimal recognition strategies. In order to validate this proposed algorithm, the target recognition software is compiled, in which 5 typical target categories and 200 data sets are combined to testify. The results show that by this proposed algorithm the correct recognition ratio is up to $99.5 \%$, which is higher than ratios attained by only using grey correlation algorithm or intuitionistic fuzzy inference algorithm, namely, that are $92.5 \%$ and $96.5 \%$, respectively. It means that by adopting this proposed algorithm the anticipated expectation can be reached. And furthermore the combination explosion issue, which possibly exists in fuzzy inference algorithm, can be effectively avoided during the time of target recognition.

Therefore the target recognition algorithm based on interval-valued intuitionistic fuzzy sets with grey correlation has comparatively better recognition results. It can accomplish the target recognition quite well and makes up the drawbacks caused by only using grey correlation or fuzzy inference method. Because of the abilities to describe and analyze the uncertain information, this method can be applied in the target recognition problems, the threat assessment problems, and the decision making problems.

In future research, the selection of upper and lower bounds of the membership and nonmembership function should be considered deeply. It is the significantly key part for the construction of target matrix. The membership and nonmembership function should be more close to the actual situation. If the target matrix is established quite well, the recognition results will be more accuracy.

\section{Competing Interests}

The authors declare that they have no competing interests.

\section{References}

[1] Y. Zhou, X. Yu, M. Cui, and X. Wang, "Radar target recognition based on multiple features fusion with Dempster-Shafer theory," in Proceedings of the IEEE 10th International Conference on Electronic Measurement and Instruments (ICEMI '11), vol. 1, pp. 243-247, Chengdu, China, August 2011.

[2] T. Tian, D. Ming, F. Jie, and B. Lei, "Fusion of multi-measures in infrared target recognition based on Dempster-Shafer evidence theory," in MIPPR: Automatic Target Recognition and Image Analysis, vol. 8003 of Proceedings of SPIE, pp. 1-8, 2011.

[3] A. Forman, D. B. Brown, and J. H. Hughen, "MUltiSensor target recognition system (MUSTRS)," in Proceedings of the 27th Asilomar Conference on Signals, Systems and Computers, vol. 1, pp. 263-267, IEEE, Pacific Grove, Calif, USA, November 1993.

[4] W. Mei, G. Shan, and C. Wang, "Model the uncertainty in target recognition using possiblized bayes' theorem," in Proceedings of the IEEE International Conference on Fuzzy Systems (FUZZ '12), pp. 1-3, Brisbane, Australia, June 2012.

[5] X. Guan, Y. He, and X. Yi, "Attribute measure recognition approach and its applications to emitter recognition," Science in China Series F: Information Sciences, vol. 48, no. 2, pp. 225-233, 2005.

[6] N. Li and F. Liu, "A SAR target recognition method based on view-aspects partitioned by fuzzy clustering," Acta Electronica Sinica, vol. 40, no. 2, pp. 394-399, 2012.

[7] X. Guan and Y. He, "A novel radar emitter recognition approach based on grey correlation analysis," Journal of System Simulation, vol. 16, no. 11, pp. 2601-2603, 2004.

[8] Y. Lin, X.-C. Si, R.-L. Zhou, and H. Yang, "Application of improved grey correlation algorithm on radiation source recognition," Journal on Communications, vol. 31, no. 8, pp. 166-171, 2010.

[9] K. T. Atanassov, "Intuitionistic fuzzy sets," Fuzzy Sets and Systems, vol. 20, no. 1, pp. 87-96, 1986.

[10] X.-H. Yuan, H.-X. Li, and C. Zhang, "The theory of intuitionistic fuzzy sets based on the intuitionistic fuzzy special sets," Information Sciences, vol. 277, no. 9, pp. 284-298, 2014.

[11] Y. Wang, Y.-J. Lei, and Y.-L. Lu, "Multiple attribute decision making method based on intuitionistic fuzzy sets," Systems Engineering and Electronics, vol. 29, no. 12, pp. 2060-2063, 2007.

[12] K. Zhang, X. Wang, C. K. Zhang, D.-Y. Zhou, and Q. Feng, "Evaluating and sequencing of air target threat based on IFE and dynamic intuitionistic fuzzy sets," Systems Engineering and Electronics, vol. 36, no. 4, pp. 697-701, 2014.

[13] S. Lu, Y.-J. Lei, W.-W. Kong, and Y. Lei, "Image registration algorithm based on intuitionistic fuzzy distance," Control and Decision, vol. 26, no. 11, pp. 1670-1674, 2011.

[14] Y. Lei, Y. J. Lei, Y. Q. Feng, and W. W. Kong, “Techniques for target recognition based on intuitionistic fuzzy reasoning," Control and Decision, vol. 26, no. 8, pp. 1163-1168, 2011.

[15] K. T. Atanassov and G. Gargov, "Interval valued intuitionistic fuzzy sets," Fuzzy Sets and Systems, vol. 31, no. 3, pp. 343-349, 1989.

[16] Z. S. Xu, "On correlation measures of intuitionistic fuzzy sets," in Intelligent Data Engineering and Automated LearningIDEAL 2006, vol. 4224 of Lecture Notes in Computer Science, pp. 16-34, Springer, Berlin, Germany, 2006.

[17] Y. J. Zhang, P. J. Ma, X. H. Su, and C. P. Zhang, "Multi-attribute decision making with uncertain attribute weight information in the framework of interval-valued intuitionistic fuzzy set," Acta Automatica Sinica, vol. 38, no. 2, pp. 220-228, 2012.

[18] H. Y. Zhang, W. X. Zhang, and C. L. Mei, "Entropy of intervalvalued fuzzy sets based on distance and its relationship with similarity measure," Knowledge-Based Systems, vol. 22, no. 6, pp. 449-454, 2009.

[19] Z. S. Xu, "On similarity measures of interval-valued intuitionistic fuzzy sets and their application to pattern recognitions," Journal of Southeast University (English Edition), vol. 23, no. 1, pp. 139-143, 2007. 


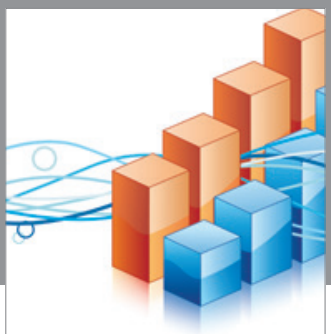

Advances in

Operations Research

vatem alat4

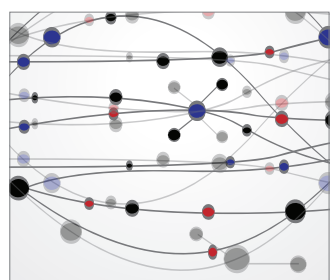

\section{The Scientific} World Journal
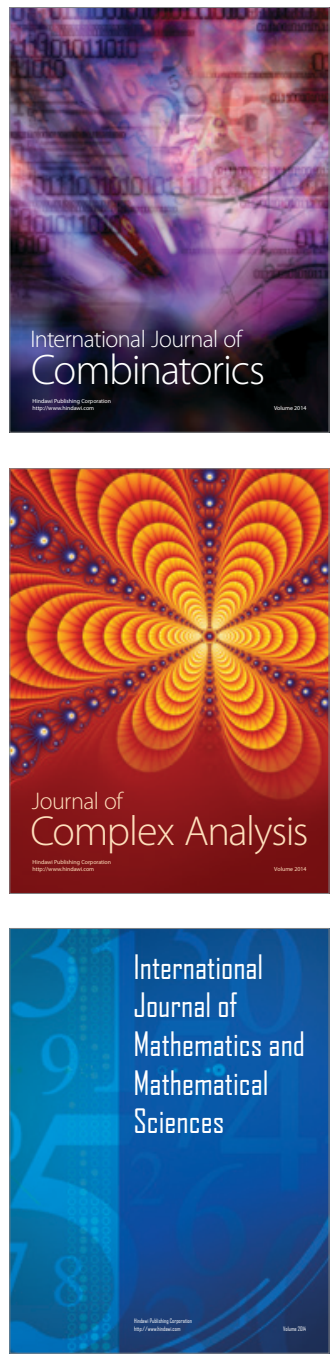
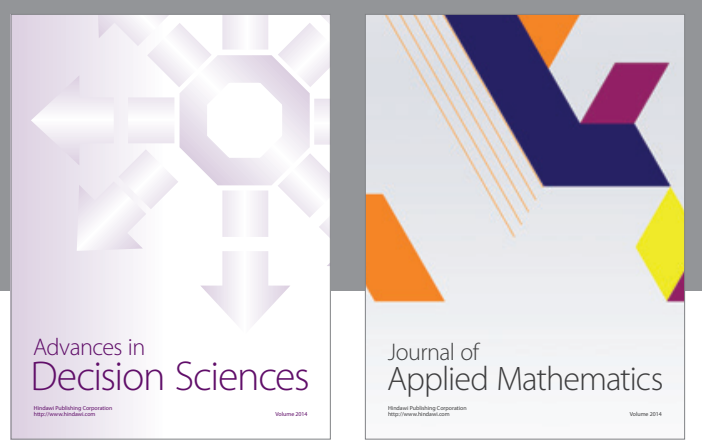

Algebra

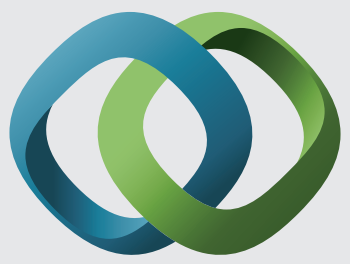

\section{Hindawi}

Submit your manuscripts at

http://www.hindawi.com
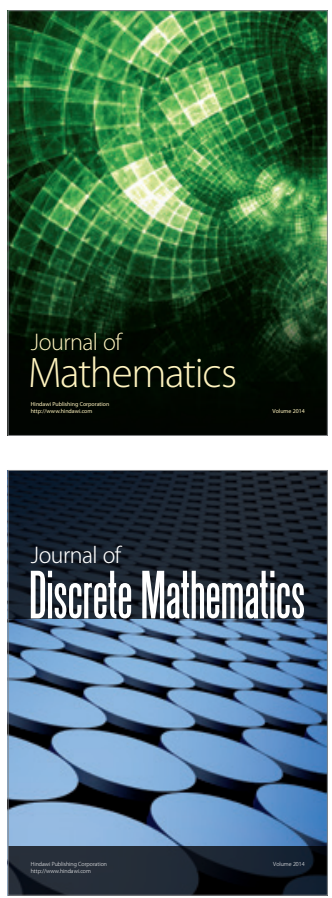

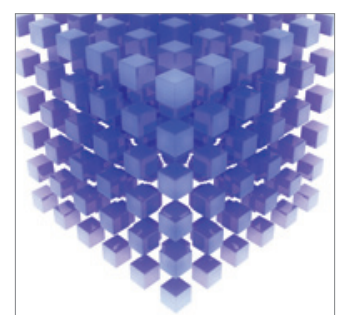

Mathematical Problems in Engineering
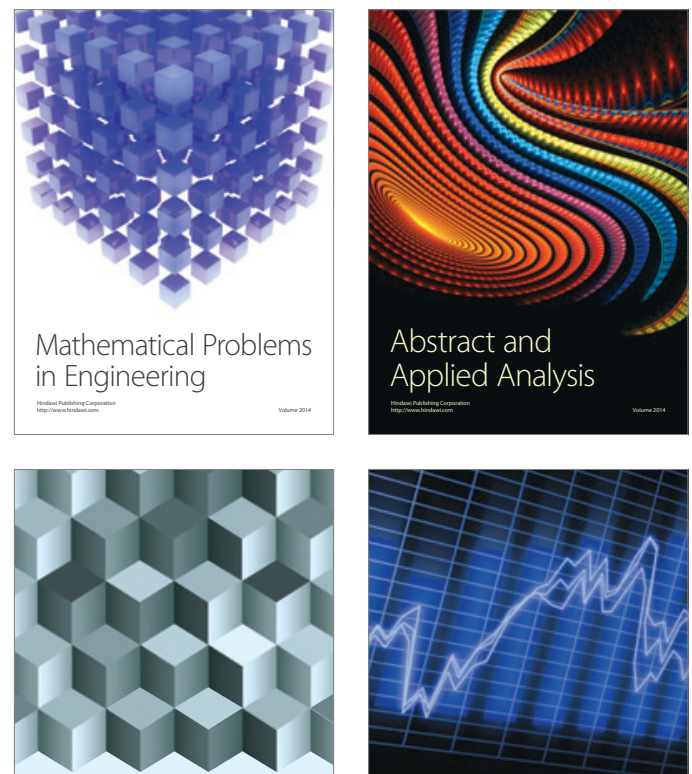

Journal of

Function Spaces

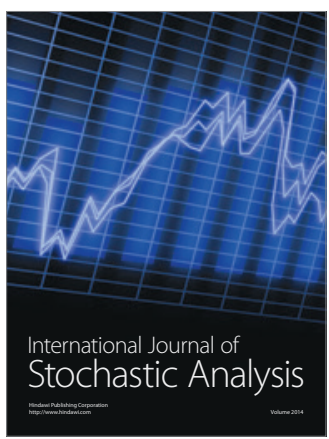

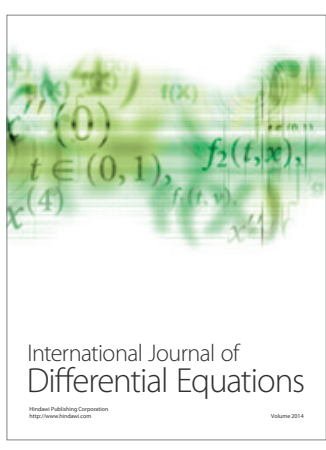
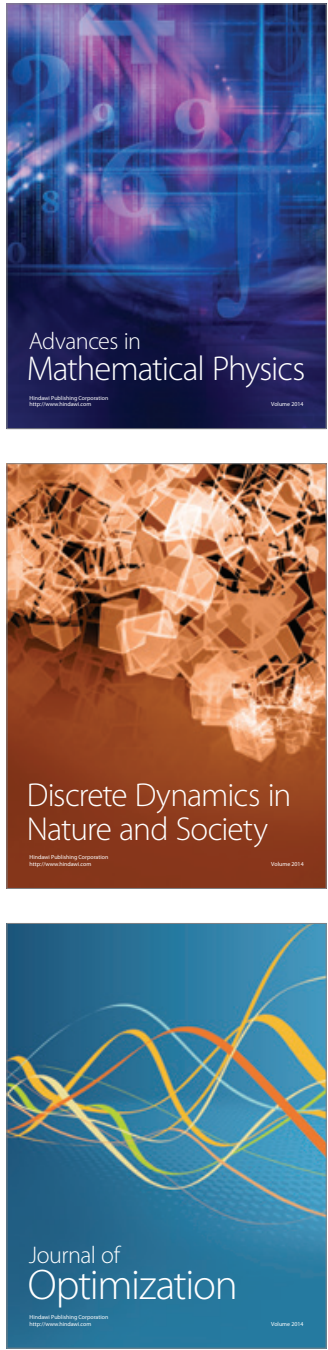